\title{
Multiphoton ionisation of atoms with soft x-ray pulses
}

\author{
M Richter ${ }^{1}$, S V Bobashev ${ }^{2}$, A A Sorokin ${ }^{1,2,3}$ and K Tiedtke ${ }^{3}$ \\ ${ }^{1}$ Physikalisch-Technische Bundesanstalt, Abbestraße 2-12, 10587 Berlin, Germany \\ ${ }^{2}$ Ioffe Physico-Technical Inst., Polytekhnicheskaya 26, 194021 St. Petersburg, Russia \\ ${ }^{3}$ Deutsches Elektronen-Synchrotron, Notkestraße 85, 22603 Hamburg, Germany \\ E-mail: mathias.richter@ptb.de
}

\begin{abstract}
The new soft x-ray Free-Electron Laser in Hamburg (FLASH) has opened the doorway to totally new experiments of materials research on nanometer and femtosecond scales. However, the mechanisms of photon-matter interaction are not well understood under the conditions of ultra-high photon intensities in conjunction with short wavelengths. In this context, we have quantitatively investigated nonlinear photoionisation of rare gas atoms at FLASH by ion mass-to-charge spectroscopy and, thus, also the limits for the application of gas-ionisation detectors for the characterisation of $\mathrm{x}$-ray lasers. By strong beam focusing, we have achieved irradiance levels beyond $10^{13} \mathrm{~W} \mathrm{~cm}^{-2}$ at about $40 \mathrm{eV}$ photon energy and up to $10^{16} \mathrm{~W} \mathrm{~cm}^{-2}$ in the Extreme Ultra-Violet (EUV) at about $90 \mathrm{eV}$. Here, surprisingly high degrees of photoionisation were observed on Xe atoms. By comparison with other rare gas targets, it emerged that the excitation of inner-shell resonances might play a significant role on the degree of atomic perturbation by the radiation field.
\end{abstract}




\section{Introduction}

The development of x-ray lasers has been a vision of the science community for many years. In 2005 it became reality when the Free-electron LASer in Hamburg (FLASH) started user operation in the soft x-ray regime [1,2]. Together with the SCSS test accelerator at Spring-8 in Japan starting in 2007 [3] and the Linac Coherent Light Source (LCLS) in Stanford starting recently [4], soft and hard x-ray laser sources are now available for the photon energy range from $20 \mathrm{eV}$ to a few $\mathrm{keV}$. These Free Electron Laser (FEL) facilities are based on Self-Amplified Spontaneous Emission (SASE) [5] and provide pulse energies up to $100 \mu \mathrm{J}$ within pulse durations of a few up to $300 \mathrm{fs}$, i.e. at peak power values of more than $1 \mathrm{GW}$. The highly intense and ultra-short soft and hard x-ray pulses allow, in particular, time-resolved studies of materials to be performed on femtosecond scales. In this context, the dynamics of chemical reactions on surfaces or within biological systems are scheduled to be investigated by microscopic snapshots using a focused FEL beam [6-9]. However, focusing increases the energy flux density, i.e. the irradiance of the laser field. At FLASH, irradiance levels up to $10^{16} \mathrm{~W}$ $\mathrm{cm}^{-2}$ have already been achieved [10]. Here, photon-matter interaction may be affected by nonlinear processes as known from optical radiation $[11,12]$.

At high photon energies, nonlinear dynamics represent, however, a largely unknown territory. It concerns, in particular, the photoionisation and photoelectron emission process which is of fundamental importance on materials research in the x-ray regime [13]. Thus, studies on photonmatter interaction at high photon energy and intensity are significant in this context for the interpretation of experimental results obtained with the current and future $\mathrm{x}$-ray lasers in fields like plasma physics, new materials, femtochemistry, and biochemical structure and dynamics. For the investigation of nonlinear processes from a fundamental point of view, such as multiphoton excitation and ionisation, gas-phase experiments are well suited, because the influence from the nearest neighbours may be detected by varying the gas pressure and can largely be suppressed by working at a sufficiently low target density. At FLASH, experiments on nonlinear processes were performed on small molecules and rare gases by ion Time-Of-Flight (TOF) mass/charge spectroscopy [10, 14-18], electron spectroscopy [19], and ion recoil momentum spectroscopy using a so-called reaction microscope [20-24]. This paper on ion TOF spectroscopy on rare gas atoms is related to the development of photon diagnostic tools for X-ray lasers that are based on gas-phase photoioinisation $[14,25-28]$. The work was performed within a scientific cooperation between the Deutsches Elektronen-Synchrotron (DESY), the Ioffe Institute St. Petersburg, and the Physikalisch-Technische Bundesanstalt (PTB), Germany's national metrology institute. 


\section{Experiment}

Figure 1 shows the different focusing geometries that were applied for our experiments. For the measurements at photon energies around $40 \mathrm{eV}$, the $15-\mu \mathrm{m}$ focus (FWHM) of the beamline BL2 at FLASH was used (figure 1a) [14]. The FEL beam was introduced into a homogeneous gas target at moderate pressures from $10^{-4}$ to $10^{-3} \mathrm{~Pa}$ to avoid any space charge or other effects from neighbouring atoms. Generated photoions were extracted towards the TOF spectrometer entrance aperture by a homogenous electric field. The aperture width of $1 \mathrm{~mm}$ along the beam ensured photoion collection exclusively from the focus region with a Rayleigh length larger than $1 \mathrm{~mm}$. The ions were detected by means of an open multiplier whose efficiency was tested to be linear in the range from 1 to $10^{6}$ particles detected per FEL pulse. The whole apparatus could be moved $\pm 2 \mathrm{~cm}$ along the FEL beam in order to vary the beam cross section and, thus, the mean irradiance within the interaction volume accepted by the TOF spectrometer.

During our measurements in the EUV at photon energies between 90 and $93 \mathrm{eV}$, the FEL beam was focused by means of spherical Mo-Si multilayer mirrors at a $20 \mathrm{~cm}$ distance from the TOF interaction volume, as shown in figure $1 \mathrm{~b}$ and figure 1c. Profiting from optical technology development in the field of EUV Lithography (EUVL), a mirror reflectance in the order of 68\% under normal incidence was achieved [29] and microfocuses of 3 to $5 \mu \mathrm{m}$ in diameter (FWHM) could be realised in backreflexion geometry $[10,17]$. As a result, the irradiance levels were higher by one to two orders of magnitude than during our measurements in the BL2 focus. In the first EUV measurement geometry, the FEL was directly focused (Figure 1b) while in the second, the BL2 focus was de-magnified by the multilayer mirror (figure 1c). In both cases, an aperture stop of $1.5 \mathrm{~mm}$ in height was used in combination with a spectrometer entrance aperture of $1.0 \mathrm{~mm}$ in height to prevent ion signals from the incident unfocused radiation. Moreover, the aperture width along the FEL beam was reduced, compared to the measurements in the BL2 focus, to about $0.3 \mathrm{~mm}$ to account for the much shorter Rayleigh length. Instead of the apparatus, the mirror was moved along the FEL beam to vary the beam cross section and the irradiance within the interaction volume. Similar geometries for photoionisation experiments on atoms at ultra-high irradiance have meanwhile been realised also at the SCSS test accelerator at Spring-8 [30].

For our experiments, emphasis was laid on the quantitative characterisation of the FEL radiation. Online detection of absolute pulse energy in the range from 1 to $100 \mu \mathrm{J}$ was performed by using GasMonitor Detectors (GMDs) [25, 26]. Their absolute calibration was performed in the PTB laboratory at the electron storage ring BESSY II and at PTB's new Metrology Light Source (MLS) in Berlin- 
Adlershof [31, 32]. FEL beam diameters in the micrometer regime were determined applying a nonlinear target depletion effect [14]. FEL pulse duration in the range from 10 to $40 \mathrm{fs}$ was estimated from the spectral distribution and confirmed by an autocorrelation technique [27, 28]. Thus, the irradiance values up to $10^{16} \mathrm{~W} \mathrm{~cm}^{-2}$ achieved could be determined traceable to SI units with $25 \%$ relative standard uncertainty.

Some of our data were normalised to the number $N$ of target atoms within the interaction volume $V$. $N$ was determined with, again, relative standard uncertainties in the order of $25 \%$ according to $N=p V / k T$ at which the (homogeneous) target gas pressure $p$ and the temperature $T$ were measured by means of a calibrated spinning rotor vacuum gauge and a calibrated Pt100 resistance thermometer, respectively. $k$ denotes the Boltzmann constant. The interaction volume $V$ was calculated, for each individual spectrometer-mirror distance, according to $V=z \pi d^{2} / 4$ with the respective FEL beam diameter $d$ (determined via target depletion [14]) and the width of the spectrometer entrance aperture along the FEL beam $z$. Due to our homogeneous ion extraction field, the latter represents a measure for the length of the interaction volume along the FEL beam.

\section{Target depletion}

Target depletion occurs if a considerable percentage of the targets within the interaction volume is ionised by a single photon pulse which may happen at the new X-ray laser sources. While for the first photons within a laser pulse, $100 \%$ of the targets are available to be photoionised, the target number may already be strongly reduced for the last photons of a pulse, which explains this type of nonlinearity. In the simplified case of a rectangular beam profile, the number $N_{i o n}$ of ions generated per pulse, normalised to the number $N$ of target atoms within the interaction volume, is described by an exponential saturation function of the photon number per pulse $N_{p h}[14]$ :

$$
\frac{N_{\text {ion }}}{N}=1-\exp \left(-N_{p h} \frac{\sigma}{A}\right)
$$

Hence, saturation due to target depletion depends on the photoionisation cross section $\sigma$ and the FEL beam cross section $A$ only. Figure 2 shows an example of such a saturation curve which we have obtained on Ne atoms in the BL2 focus of FLASH (figure 1a) at the photon energy of $38.0 \mathrm{eV}$. The effect was used as a new method to derive FEL beam diameters and, from that, focus size and beam waist $[14,33]$. For this purpose, the absolute photon number per pulse was measured with the aid of a calibrated GMD, and the measured saturation curves were fitted by equation 1, using the known onephoton single ionisation cross section of $\mathrm{Ne} \sigma(38.0 \mathrm{eV})=(8.6 \pm 0.3) \times 10^{-18} \mathrm{~cm}^{2}$ [34]. 


\section{Sequential and simultaneous multiphoton ionisation}

If a considerable percentage of the targets within the interaction volume is ionised by a single photon pulse, the generated ions represent a new sort of target which can be further ionised within the same pulse. In particular, sequences of photoionisation processes may occur as long as the photon pulse duration is short enough, so that the atomic and ionic targets do not drop out of the interaction volume:

$$
\mathrm{X} \stackrel{\text { 1st photon }}{\longrightarrow} \mathrm{X}^{1+} \stackrel{\text { 2nd photon }}{\longrightarrow} \mathrm{X}^{2+} \quad \ldots
$$

This type of sequential multiphoton ionisation is restricted to photon energies above the respective ionisation thresholds. As an example, the sequential two-photon double ionisation was studied at FLASH, firstly on molecular nitrogen at the photon energy of $38.0 \mathrm{eV} \mathrm{[15]} \mathrm{and} \mathrm{later} \mathrm{on} \mathrm{Ne}$ atoms in the same spectral range [16]. These experiments have demonstrated that (soft) X-ray lasers like FLASH allow the investigation of ions that are prepared by the highly intense photon pulses themselves, i.e. as intermediate targets from initial atomic or molecular targets in a first step of a photoionisation sequence according to equation 2. This idea was applied to determine photoionisation cross sections for different ion species, e.g. of Ne: the one-photon single ionisation cross section of $\mathrm{Ne}^{+} \rightarrow \mathrm{Ne}^{2+}$ (second step in figure 3(b)) and the simultaneous two-photon single ionisation of $\mathrm{Ne}^{2+} \rightarrow$ $\mathrm{Ne}^{3+}$ (third step in figure 3(c)), at $42.8 \mathrm{eV}$ photon energy, and the two-photon single ionisation cross section of $\mathrm{Ne}^{+} \rightarrow \mathrm{Ne}^{2+}$ at $38.4 \mathrm{eV}$ photon energy (second step in figure 3(d)) [16]. The latter process was, however, probably affected by a $2 p \rightarrow 4 s$ resonance at $38.2 \mathrm{eV}$ [35] so that it may be interpreted as a two step rather than a single step two-photon ionisation. Resonant multiphoton processes via $\mathrm{Ne}^{+}$ $2 p \rightarrow \mathrm{n} \ell$ resonance states were particularly studied also between 41 and $42 \mathrm{eV}$ photon energy [18].

In table 1 , absolute cross section values are listed that were derived from the dependence of the $\mathrm{Ne}^{2+}$ to $\mathrm{Ne}^{+}$and $\mathrm{Ne}^{3+}$ to $\mathrm{Ne}^{2+}$ ion-yield ratios on photon exposure $N_{p h} / A$ and, with $\hbar \omega$ and $\Delta t$ denoting photon energy and FEL pulse duration, irradiance $E=\left(N_{p h} / A\right) \cdot(\hbar \omega / \Delta t)$. For this purpose, the data points were fitted, as shown in figure 4, by applying the general power law of perturbation theory for the rate of nphoton processes:

$$
\dot{N}^{(\mathrm{n})}=N \sigma^{(\mathrm{n})}\left(\frac{E}{\hbar \omega}\right)^{\mathrm{n}}
$$

with the respective generalised n-photon ionisation cross sections $\sigma^{(\mathrm{n})}$ and the number $N$ of (intermediate) targets within the interaction volume. 
Table 1 also comprises the value of $(1.6 \pm 0.6) \times 10^{-52} \mathrm{~cm}^{4} \mathrm{~s}$ for the simultaneous two-photon double ionisation cross section of $\mathrm{He} \rightarrow \mathrm{He}^{2+}$ at $42.8 \mathrm{eV}$ photon energy, evaluated from the measured $\mathrm{He}^{2+}$ to $\mathrm{He}^{+}$ion-yield ratio which is shown in figure 4, too [16]. The simultaneous two-photon double ionisation of $\mathrm{He}$ has been a showcase of considerable interest during the last few years demonstrated by numerous theoretical papers (see, e.g., [36-44] and references therein). A satisfying calculation of the corresponding generalised cross section from first principles, however represents a challenge, because the results differ by almost one order of magnitude as shown in figure 5 . The experimental data, estimated from a high-harmonic generation (HHG) experiment performed at $41.8 \mathrm{eV}[45,46]$ and resulting from our study performed at $42.8 \mathrm{eV}$ at FLASH [16], lie within the two main groups of theoretical values. Good agreement with the experimental results has been achieved, so far, in the more recent work by Ivanov and Kheifets [43].

The strength of simultaneous multiphoton ionisation depends on powers of irradiance $E$ and, hence, also on the pulse duration $\Delta t$. The latter may, therefore, be experimentally determined by means of an autocorrelation technique [45]. Splitting an FEL pulse with the temporal pulse shape $f(t)$ into two parts with the irradiance amplitudes $E_{1}$ and $E_{2}$ and a temporal delay of $t^{\prime}\left(E(t)=E_{l} f(t)+E_{l} f\left(t-t^{\prime}\right)\right)$, one obtains, according to equation 3 , for a simultaneous two-photon process:

$$
\frac{N^{(2)}\left(t^{\prime}\right)}{N^{(2)}\left(t^{\prime} \rightarrow \infty\right)}-1 \sim \int_{-\infty}^{+\infty} f(t) f\left(t-t^{\prime}\right) d t
$$

from which the pulse shape function $f(t)$ and, with that, the pulse duration may be derived. Figure 6 shows the result of a corresponding autocorrelation experiment performed at FLASH on the simultaneous two-photon double ionisation of He at $51.8 \mathrm{eV}$ photon energy [28]. The Gaussian fit to the data points yields an autocorrelation width of $39 \mathrm{fs}$ and the pulse duration of $(28 \pm 5) \mathrm{fs}$.

\section{Multiphoton ionisation and strong-field phenomena in the EUV}

Our FLASH results of nonlinear photoionisation presented in sections 3 and 4 can be well explained in terms of target depletion and multiphoton schemes. In the EUV, however, exceptional behaviour of light-matter interaction was found. With the aid of a high-quality industrial grade spherical Si/Mo multilayer mirror, of the type used in EUV-lithography [29], we have realised focal spots of 3 to $5 \mu \mathrm{m}$ in diameter at photon energies from 90 to $93 \mathrm{eV}$ and, hence, irradiance levels up to $10^{16} \mathrm{~W} \mathrm{~cm}^{-2}[10$, 17]. The experiments were performed on rare gases in the set-up geometries shown in figures $1 \mathrm{~b}$ and 1c. In the case of $\mathrm{Xe}$, the situation is rather complex. Figure 7 shows the nonlinear appearance of 
higher charge states with increasing EUV irradiance $E$ obtained in the set-up geometry of figure $1 \mathrm{~b}$ at $93 \mathrm{eV}$ photon energy [10]. At $E=7.8 \times 10^{15} \mathrm{~W} \mathrm{~cm}^{-2}, \mathrm{Xe}^{21+}$ occurs whose generation, starting from neutral $\mathrm{Xe}$, requires a total energy of at least $5 \mathrm{keV}$. Plasma effects like electron impact from neighbouring atoms can be excluded, because the spectra do not depend on the target density. This means that almost $60 \mathrm{EUV}$ photons at the energy of $93 \mathrm{eV}$ must have been absorbed by an individual atom within the estimated FLASH pulse duration in the EUV of 10 to $20 \mathrm{fs}$.

In a first theoretical study on our Xe results, the high degree of photoionisation was explained by a sequence of one- and multiphoton ionisation processes according to equation 2 in which an ion created in a preceding step represents the target for a subsequent step [47]. This approach has to consider at least 19 steps to generate $\mathrm{Xe}^{21+}$ from atomic $\mathrm{Xe}$ which is related to 19 coupled differential rate equations with 19 individual multiphoton ionisation cross sections. The latter were not calculated from first principles but obtained through a technique of scaling, with occasional adjustment to the experimental data. The work represents, thus, rather a theoretical interpretation of the experimental results than an ab initio calculation.

On the other hand, in the spectral range of optical radiation, strong-field phenomena are often described also by so-called non-perturbative theories [11, 12]. Their applicability depends on the ponderomotive energy $U_{p}$ :

$$
U_{p} / \mathrm{eV} \approx 1.44 \times \frac{E /\left(10^{13} \mathrm{~W} \mathrm{~cm}^{-2}\right)}{(\hbar \omega / \mathrm{eV})^{2}}
$$

which is the quiver energy transferred to a quasi-free electron by the oscillating field of an electromagnetic wave. However, $U_{p}$ decreases with the square of the photon energy $\hbar \omega$ and is, thus, by far too low to explain multiphoton ionisation in the EUV at $E=7.8 \times 10^{15} \mathrm{~W} \mathrm{~cm}^{-2}$.

In order to obtain a deeper insight into the photoionisation process in the EUV at irradiance levels beyond $10^{15} \mathrm{~W} \mathrm{~cm}^{-2}$, we have, in a further experimental study, compared Xe with $\mathrm{Ne}$, Ar, and $\mathrm{Kr}$ [17]. The gases were investigated at FLASH in the set-up geometry of figure 1c under equivalent conditions at $90.5 \mathrm{eV}$ photon energy. Figure 9 shows the corresponding ion TOF spectra. At irradiance levels in the range from 1.5 to $1.8 \times 10^{15} \mathrm{~W} \mathrm{~cm}^{-2}$, the highest charge state observable with our experiments is $7+$ for $\mathrm{Ne}, \mathrm{Ar}$, and $\mathrm{Kr}$ but $14+$ in the case of $\mathrm{Xe}$.

The particular behaviour of Xe observed when irradiated by ultra-high intensities in the EUV is also summarised in table 2. The minimum number $\mathrm{n}_{\min }$ of EUV photons with $90.5 \mathrm{eV}$ photon energy, 
which must have been absorbed within a single FEL pulse by an individual atom to deliver the amount of energy for the highest charge state observable, is listed in the $4^{\text {th }}$ row. It strongly varies from $\mathrm{n}_{\min }=$ 5 for $\mathrm{Kr}$ and $\mathrm{Ar}, \mathrm{n}_{\min }=8$ for $\mathrm{Ne}$, and $\mathrm{n}_{\min }=22$ for $\mathrm{Xe}$. This is obviously reflected by the respective one-photon ionisation cross section values for $90.5 \mathrm{eV}$ photons which are listed in the last row of table 2. In the case of $\mathrm{Xe}$, the photoionisation cross section is strongly enhanced by the so-called giant $4 d \rightarrow$ $\varepsilon f$ continuum resonance and amounts to $24 \times 10^{-18} \mathrm{~cm}^{2}$.

On the role of the $4 d$ giant resonance for the interpretation of our results, a controversial discussion has started recently [47]. In comparison to the other rare gases, it is a particular feature of Xe and arises in the photon energy range from about 85 to $115 \mathrm{eV}$ [48]. For many years, this resonance has represented a prime example of the impact of electron correlation on inner-shell photoionisation at low irradiance $[49,50]$. The idea that the strength and nature of this resonance may also drive, at high irradiance, direct multiphoton excitation from the inner $4 d$ shell constitutes the core of the controversy. However, future theoretical work and more advanced experiments may decide between the two proposals: (1) The $4 d \rightarrow \varepsilon f$ resonance plays a role just at the beginning of a sequence of one- and multiphoton ionisation processes by one-photon single-electron $4 d$ excitation with subsequent Auger decay [47] or (2) the strong electron correlation within the $4 d$ shell leads to a collective resonance resulting, at high irradiance, to multiphoton multiple-electron excitation in the $4 d$ shell with subsequent decay [17].

\section{Conclusion}

From our gas-phase experiments performed in focused beams at FLASH, it emerges out that the photoionisation of matter is strongly affected by inner-atomic nonlinear behaviour at irradiance levels beyond $10^{13} \mathrm{~W} \mathrm{~cm}^{-2}$. Most of the results we have obtained on rare gases can be well interpreted in terms of sequences of one-photon and multiphoton processes. How to explain the high charge states of up to $21+$ observed at EUV irradiance levels between $10^{15}$ and $10^{16} \mathrm{~W} \mathrm{~cm}^{-2}$ on $\mathrm{Xe}$ atoms remains, however, still an open question. A perturbative approach seems to be challenging in this context, because nearly 50 photons have to be considered interacting with a single atom within 10 to 20 fs. Non-perturbative theories based on the ponderomotive motion of quasi-free electrons as developed for optical femtosecond pulses are not applicable either. Thus, further theoretical investigations, in particular, into the role of inner-shell (giant) resonances and collective effects on photoionisation in the high-intensity short wavelength regime, seem to be necessary. From the experimental side, the application of electron and fluorescence spectroscopy at FLASH, at the SCSS test accelerator at 
Spring-8, at LCLS, or the upcoming XFELs in Japan and Hamburg, will certainly make a major contribution in this context.

The investigation of multiphoton processes on atoms at the new x-ray laser sources has at least two interesting perspectives. The first concerns the influence of nonlinear processes on photon-matter interaction and the mechanisms of x-ray induced destruction of materials by electronic excitation, in general. This aspect is significant for all x-ray laser applications in various fields of materials research. The second concerns a fascinating new field of fundamental science at the limits of a quantum mechanical and a semi-classical description of photoionisation.

\section{Acknowledgement}

We thank the FLASH team for the very successful operation of the FEL; we also thank T. Feigl, J. Feldhaus, M. Martins, R. Mitzner, J. Schneider, B. Sonntag, G. Ulm, and H. Zacharias for continuous support and many helpful discussions; support by the Deutsche Forschungsgemeinschaft (DFG) is gratefully appreciated. 


\section{References}

[1] Ayvazyan V et al 2005 Eur. Phys. J. D 37297

[2] Ackermann W et al 2007 Nat. Photonics 1336

[3] Shintake T et al 2008 Nature Photon. 2555

[4] ScienceNOW, April 21, 2009

[5] Ayvazyan V et al 2002 Eur. Phys. J. D 20149

[6] O'Shea P G and Freund H P 2001 Science 2921583

[7] Kapteyn H C and Ditmire T 2002 Nature 420467

[8] Feldhaus J, Arthur J and Hastings J B 2005 J. Phys. B: At. Mol. Opt. Phys. 38 S799

[9] Bostedt C et al 2009 Kai Siegbahn Memorial Volume of Nucl. Instr. and Meth. A 601108

[10] Sorokin A A et al 2009 Phys. Rev. Lett. 99213002

[11] Protopapas M, Keitel C H and Knight P L 1997 Rep. Prog. Phys. 60389

[12] Delone N and Krainov V 2000 Multiphoton Processes in Atoms (New York: Springer)

[13] Siegbahn K, 1997 X-rays in Natural and Life Sciences, in: Haase A, Landwehr G and Umbach E (Eds.) Röntgen Centennial (Singapore: World Scientific) 397

[14] Sorokin A A et al 2006 Appl. Phys. Lett. 89221114

[15] Sorokin A A et al 2006 J. Phys. B: At. Mol. Opt. Phys. B 39 L299

[16] Sorokin A A et al 2007 Phys. Rev. A 75 051402(R)

[17] Richter M, et al 2009 Phys. Rev. Lett. 102, 163002

[18] Martins M et al 2009 Phys. Rev. A 80023411

[19] Nagasono M et al 2007 Phys. Rev. A 75 051406(R)

[20] Moshammer R et al 2007 Phys. Rev. Lett. 98203001

[21] Rudenko A et al 2008 Phys. Rev. Lett. 101073003

[22] Jiang Y H 2009 Phys. Rev. Lett. 102123002

[23] Kurka M et al 2009 J. Phys. B: At. Mol. Opt. Phys. B 42141002

[24] Jiang Y H et al 2009 J. Phys. B: At. Mol. Opt. Phys. B 42134012

[25] Richter M et al 2003 Appl. Phys. Lett. 832970

[26] Tiedtke K et al 2008 J. Appl. Phys. 103, 094511

[27] Mitzner R et al 2008 Opt. Express 16, 19909

[28] Mitzner R et al 2009 Phys. Rev. A 80, 025402

[29] Feigl T, Yulin S, Benoit N and Kaiser N 2006 Microelectron. Eng. 83703

[30] Motomura K et al 2009 J. Phys. B: At. Mol. Opt. Phys. B 42221003

[31] Beckhoff B et al 2009 Phys. Status Solidi B 2461415

[32] Saito N et al 2010 Metrologia 4721

[33] Wellhöfer M et al 2007 J. Opt. A 9, 749 
[34] Bizau J M and Wuilleumier F J 1995 J. Electron Spectrosc. Relat. Phenom. 71205

[35] Kramida A E et al 2006 Phys. Scr. 74156

[36] Feng L and van der Hart H W 2003 J. Phys. B 36 L1

[37] Laulan S, Bachau H 2003 Phys. Rev. A 68013409

[38] Foumouo E, Kamta G L, Edah G and Piraux B 2006 Phys. Rev. A 74063409

[39] Nikolopoulos L A A and Lambropoulos P 2007 J. Phys. B 401347

[40] Horner D A et al 2007 Phys. Rev. A $76030701(\mathrm{R})$

[41] Ivanov I A and Kheifets A S 2008 Phys. Rev. A 75033411

[42] Feist J et al 2008 Phys. Rev. A 77043420

[43] Ivanov I A and Kheifets A S 2008 J. Phys. B: At. Mol. Opt. Phys. 41095002

[44] Piraux B, Foumouo E, Antoine P and Bachau H 2008 J. Phys. Conf. Series 141012013

[45] Nabekawa Y et al 2005 Phys. Rev. Lett. 94043001

[46] Hasegawa H et al 2005 Phys. Rev. A 71023407

[47] Makris M G, Lambropoulos P and Mihelic A 2009 Phys. Rev. Lett. 102033002

[48] Becker U and Shirley D A (Eds.) 1996 VUV and Soft X-Ray Photoionization (New York: Plenum Press) and references therein

[49] Connerade J P, Esteva J M and Karnatak R C 1987 Giant Resonances in Atoms, Molecules, and Solids (New York: Plenum Press) and references therein

[50] Amusia M Ya and Connerade J P 2000 Rep. Prog. Phys. 6341 
Table 1. Cross section values measured for different one- and two-photon ionisation processes on atomic, molecular, and ionic targets [15, 16, 18].

\begin{tabular}{lc}
\hline Photoionisation process & Cross section \\
\hline One-photon single ionisation/dissociation of $\mathrm{N}_{2}^{+}$at $38.0 \mathrm{eV}$ photon energy & $(1.0 \pm 0.3) \times 10^{-18} \mathrm{~cm}^{2}$ \\
One-photon single ionisation of $\mathrm{Ne}^{+}$at $41.2 \mathrm{eV}$ photon energy & $(3.1 \pm 1.1) \times 10^{-18} \mathrm{~cm}^{2}$ \\
One-photon single ionisation of $\mathrm{Ne}^{+}$at $42.8 \mathrm{eV}$ photon energy & $(7.0 \pm 1.0) \times 10^{-18} \mathrm{~cm}^{2}$ \\
Two-photon single ionisation of $\mathrm{Ne}^{+}$at $38.4 \mathrm{eV}$ photon energy & $(1.6 \pm 0.6) \times 10^{-50} \mathrm{~cm}^{4} \mathrm{~s}$ \\
Two-photon single ionisation of $\mathrm{Ne}^{2+}$ at $42.8 \mathrm{eV}$ photon energy & $(3.0 \pm 1.2) \times 10^{-51} \mathrm{~cm}^{4} \mathrm{~s}$ \\
Two-photon double ionisation of $\mathrm{He}$ at $42.8 \mathrm{eV}$ photon energy & $(1.6 \pm 0.6) \times 10^{-52} \mathrm{~cm}^{4} \mathrm{~s}$ \\
\hline
\end{tabular}

Table 2. Highest charge state $\mathrm{q}_{\text {max }}^{+}$observed at irradiance levels in the range from 1.5 to $1.8 \times 10^{15} \mathrm{~W} \mathrm{~cm}^{-2}$, ionisation energy $I$ required to reach this state starting from the atomic ground state, corresponding minimum number $n_{\min }$ of EUV photons of $90.5 \mathrm{eV}$ photon energy which must have been absorbed within a single FEL pulse by an individual atom to deliver this amount of energy, and one-photon ionisation cross section $\sigma$ at $90.5 \mathrm{eV}$ photon energy for the rare gases $\mathrm{Ne}, \mathrm{Ar}, \mathrm{Kr}$, and Xe, respectively [17].

\begin{tabular}{ccccc}
\hline $\mathrm{Gas}$ & $\mathrm{q}_{\max }^{+}$ & $I / \mathrm{eV}$ & $\mathrm{n}_{\min }$ & $\sigma / 10^{-18} \mathrm{~cm}^{2}$ \\
\hline $\mathrm{Ne}$ & $7+$ & 715 & 8 & 4.4 \\
$\mathrm{Ar}$ & $7+$ & 434 & 5 & 1.35 \\
$\mathrm{Kr}$ & $7+$ & 383 & 5 & 0.55 \\
$\mathrm{Xe}$ & $14+$ & 1930 & 22 & 24 \\
\hline
\end{tabular}




\section{Figure captions}

Figure 1. Schematic diagrams of the experimental set-ups as used for ion Time-Of-Flight (TOF) spectroscopy on atoms at FLASH: (a) in the focus of beamline BL2 [14-16]; (b) in the focus of a spherical EUV multilayer mirror [10]; (c) in the de-magnified BL2 focus using a spherical EUV multilayer mirror [17].

Figure 2. Number of $\mathrm{Ne}^{+}$ions generated per FEL pulse from $\mathrm{Ne}$ atoms measured as a function of the photon number per pulse at the photon energy of $38.0 \mathrm{eV}$ (open symbols). The solid line represents a fit curve according to equation 1 . The dashed line depicts a linear dependence for comparison [14].

Figure 3. Energy diagram and photoionisation schemes for neon: one-photon single ionisation (a), sequential two-photon double ionisation (b), and four-photon triple ionisation (c) by $42.8 \mathrm{eV}$ photons and three-photon double ionisation (d) by $38.4 \mathrm{eV}$ photons. The dashed lines indicate virtual and/or resonant states.

Figure 4. Ion-yield ratios as a function of mean pulse irradiance and photon exposure measured at 42.8 and $38.4 \mathrm{eV}$ photon energy [16]. The lines represent fit curves according to equation 3.

Figure 5. Two-photon double ionisation cross section of He as measured at FLASH [16] and using an HHG source [45, 46]. Some theoretical predictions are shown for comparison: $\bullet \bullet \bullet[39], \bullet \bullet \bullet[38], \triangle \triangle \triangle$ [43], $\square \square \square[37], \diamond \diamond \diamond[36], \circ \circ \circ[39]$.

Figure 6. Double ionisation signal of He measured at $51.8 \mathrm{eV}$ photon energy as a function of the time delay between two autocorrelator pulses (dots). The solid line represents a Gaussian function with 39 fs FWHM yielding a pulse duration at FLASH of $(28 \pm 5)$ fs. The dashed line represents a simulation with a three-pulse structure for the temporal pulse shape of the FEL [27, 28].

Figure 7. Ion time-of-flight (TOF) mass/charge spectra of $\mathrm{Xe}$ taken at the photon energy of $93 \mathrm{eV}$ and different pulse irradiance levels [10].

Figure 8. Relative $\mathrm{Xe}^{\mathrm{q}+}$ ion signal intensities as a function of the peak irradiance for different charge states $1 \leq \mathrm{q}$ $\leq 15$ at the photon energy of $93 \mathrm{eV}$. The symbols show the experimental data obtained at FLASH [10] whose initial slopes are shown by the dashed lines (for $\mathrm{q} \leq 9$ ). The solid lines represent model calculations within the framework of a sequential multiphoton scheme [47]. 
(a) ion TOF spectrometer

FEL beam $\longrightarrow$

(b)

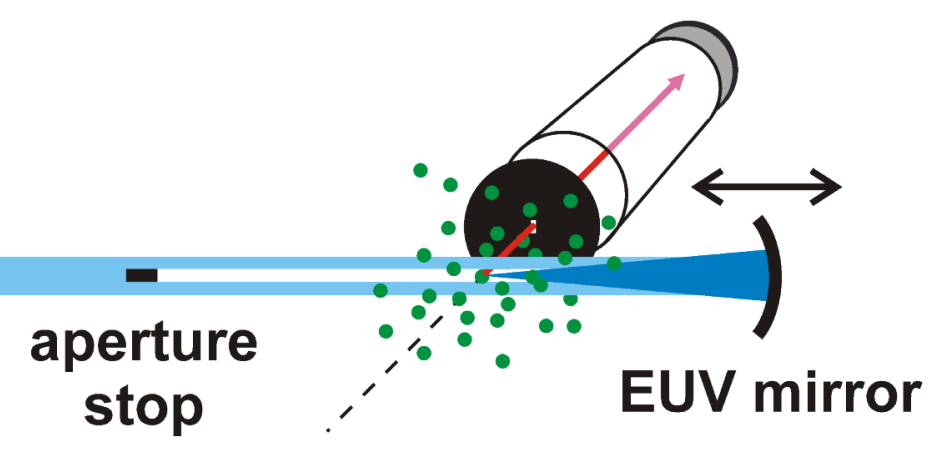

(c)

BL2 focus

$x$

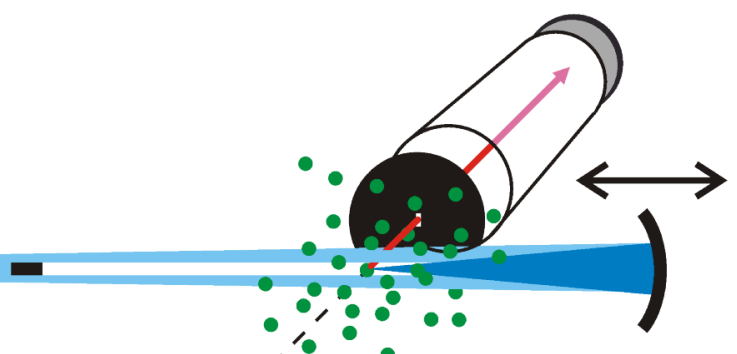

Figure 1 (Fig01_Setups.png) 


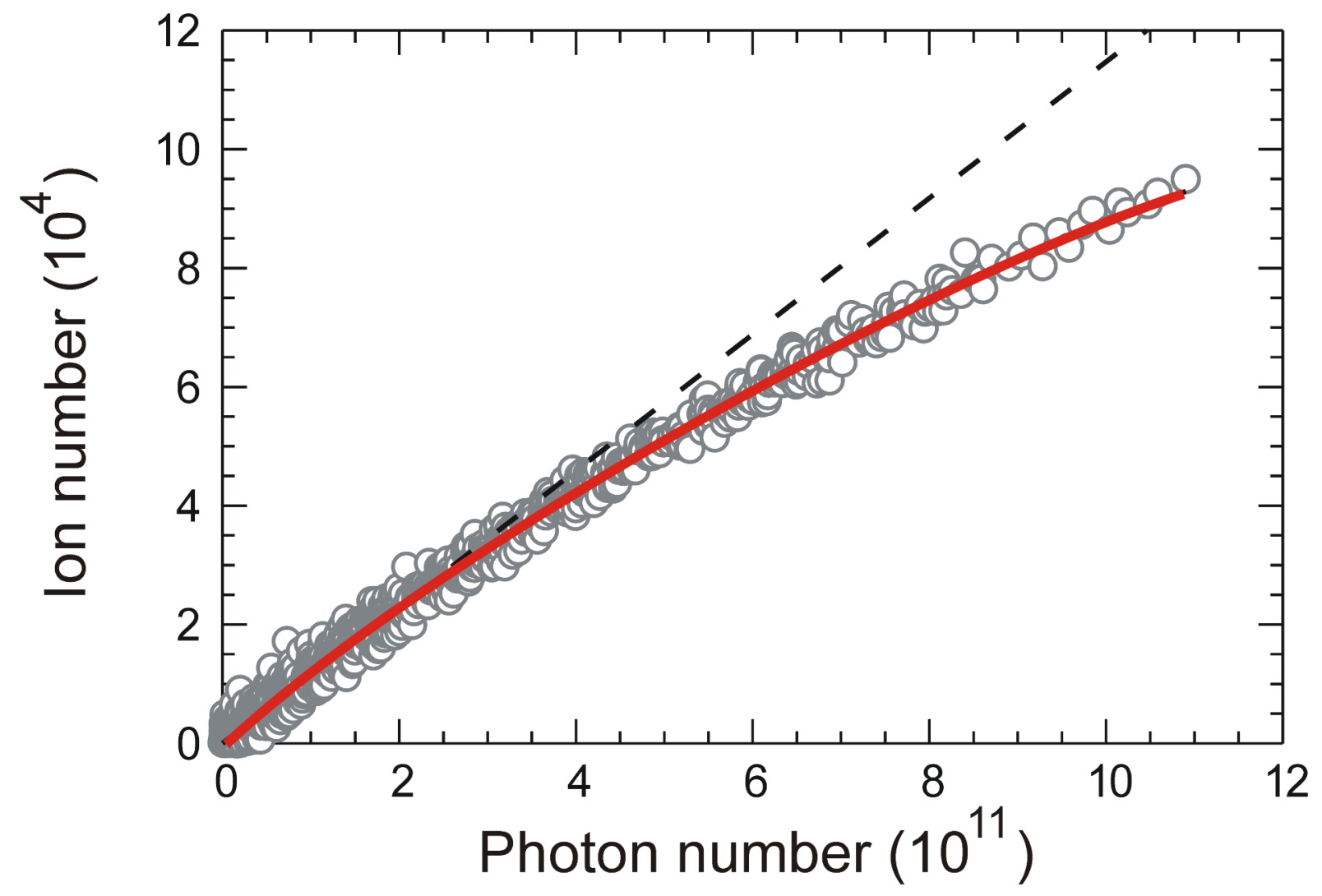

Figure 2 (Fig02_NeonSat.png) 
Neon Energy / eV

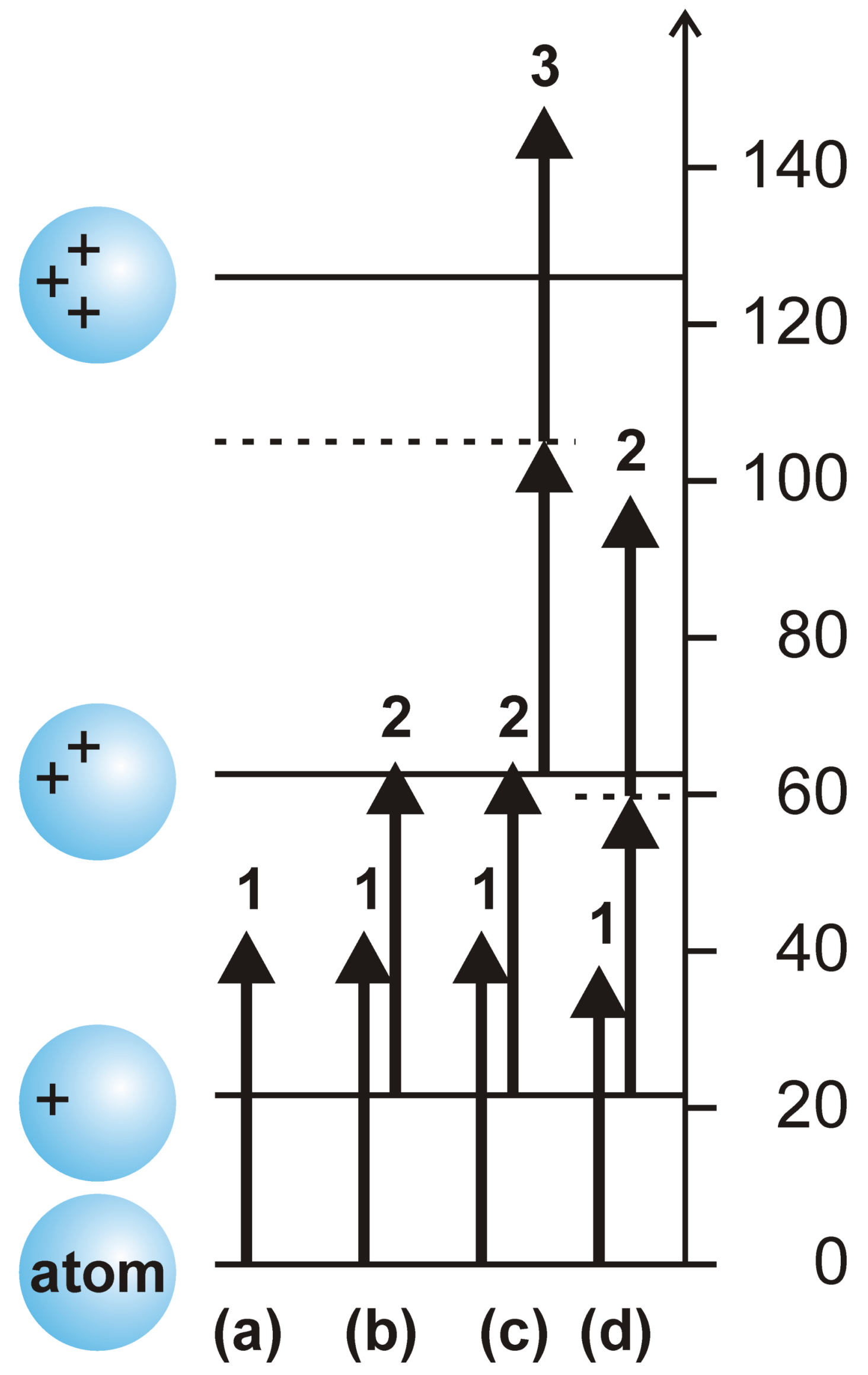

Figure 3 (Fig03_NeonEneregieScheme.png) 


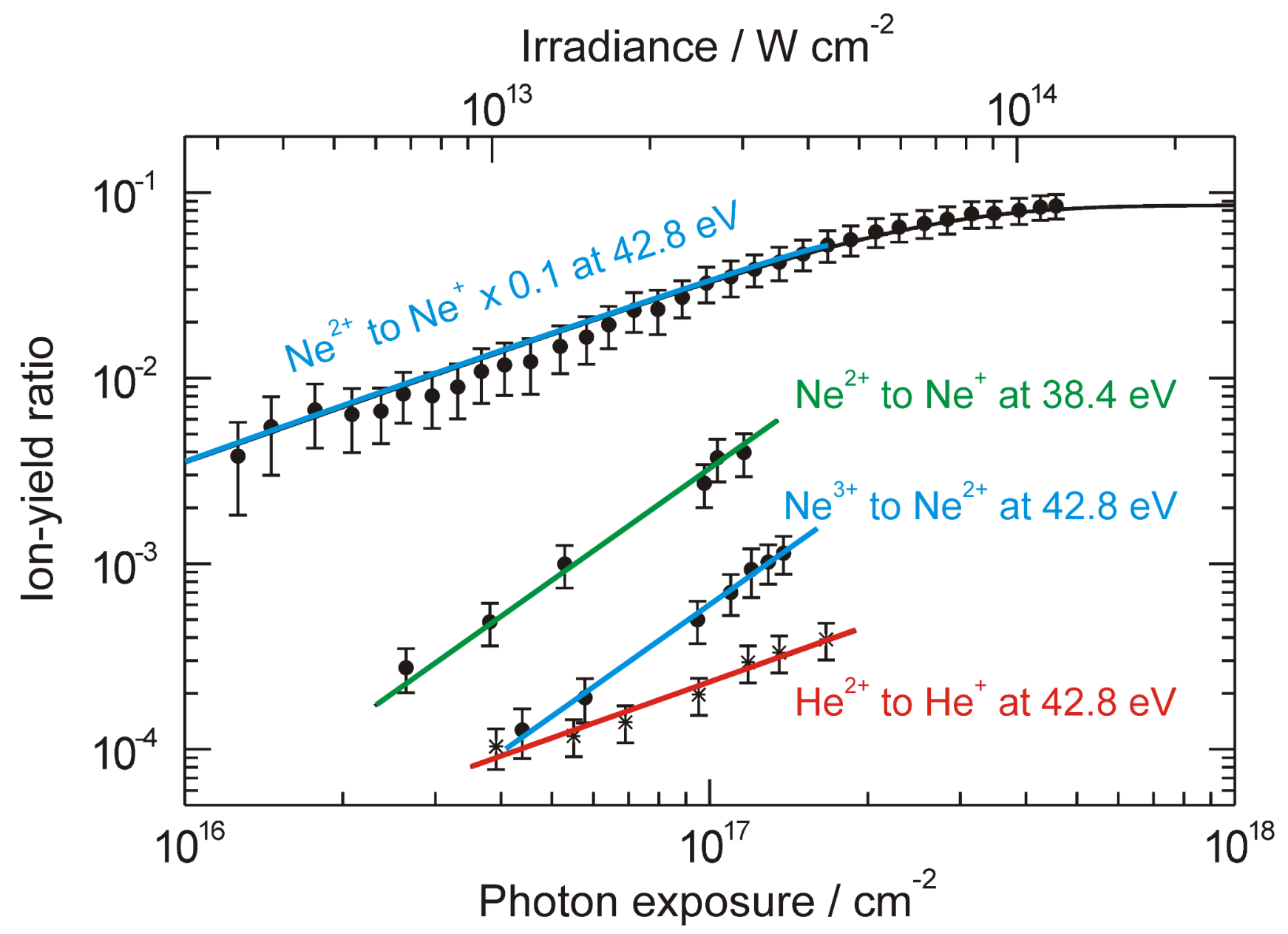

Figure 4 (Fig04_HeNeIntDep.png) 


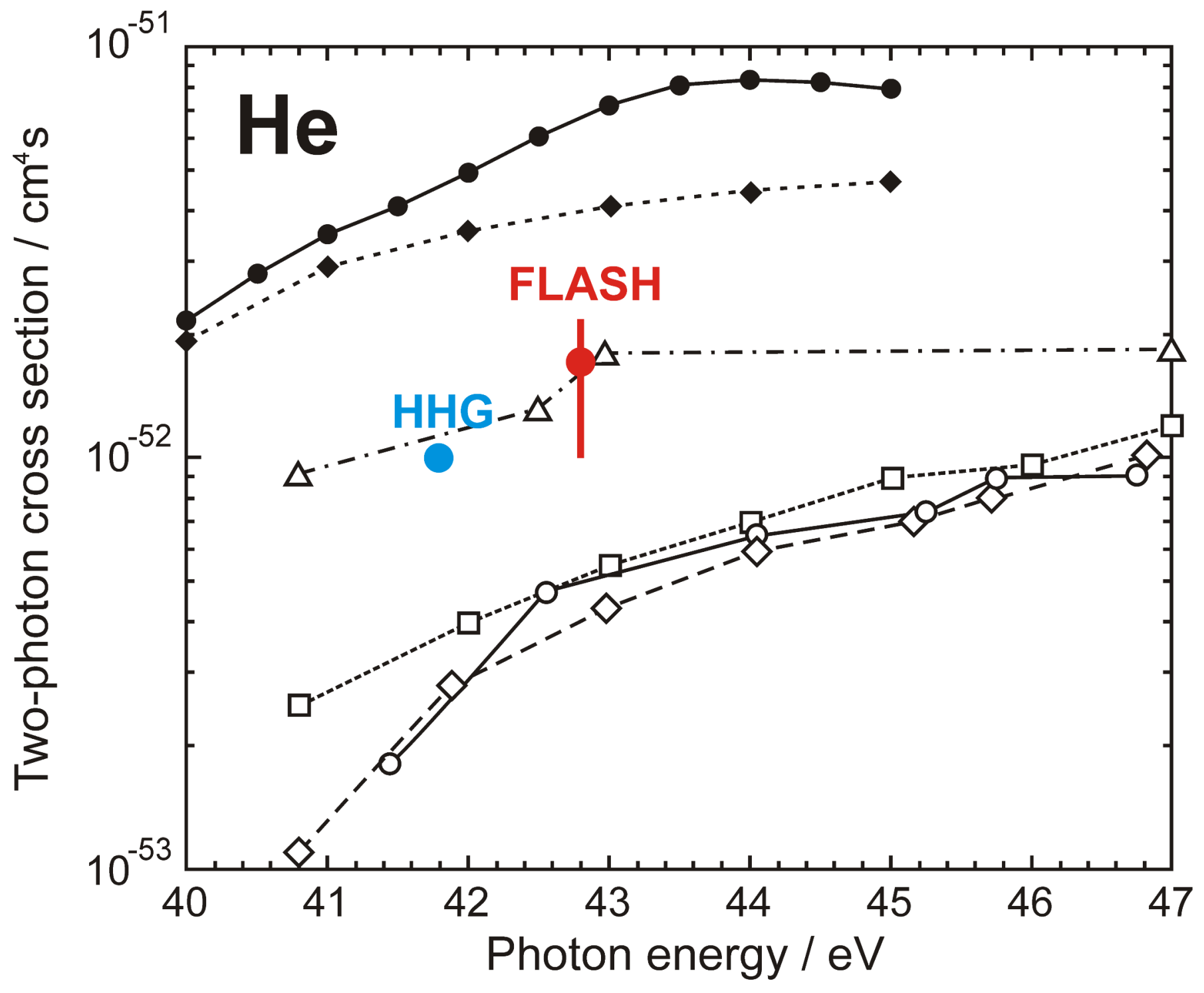

Figure 5 (Fig05_HeCrossSection.png) 


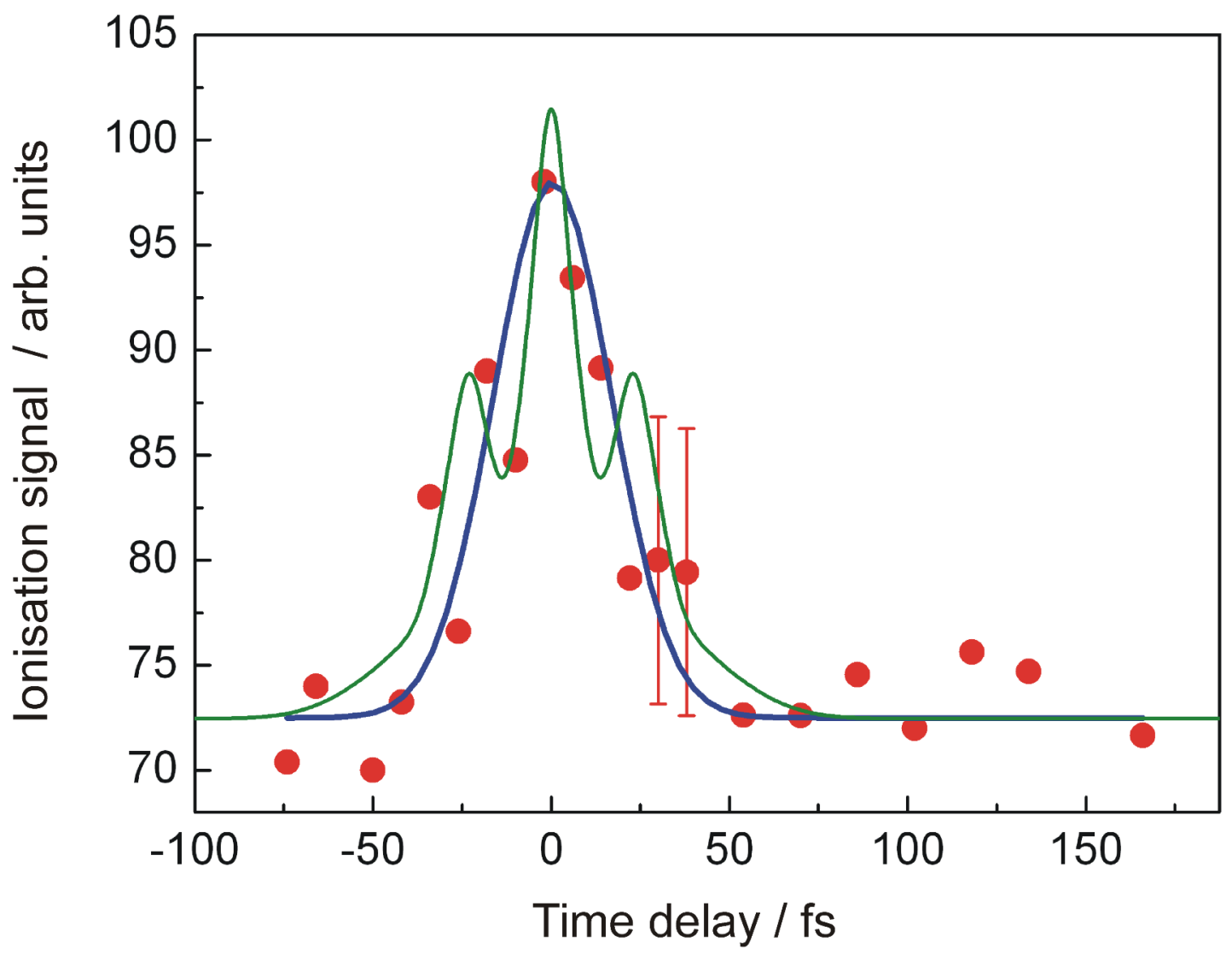

Figure 6 (Fig06_HeAutocorr.png) 


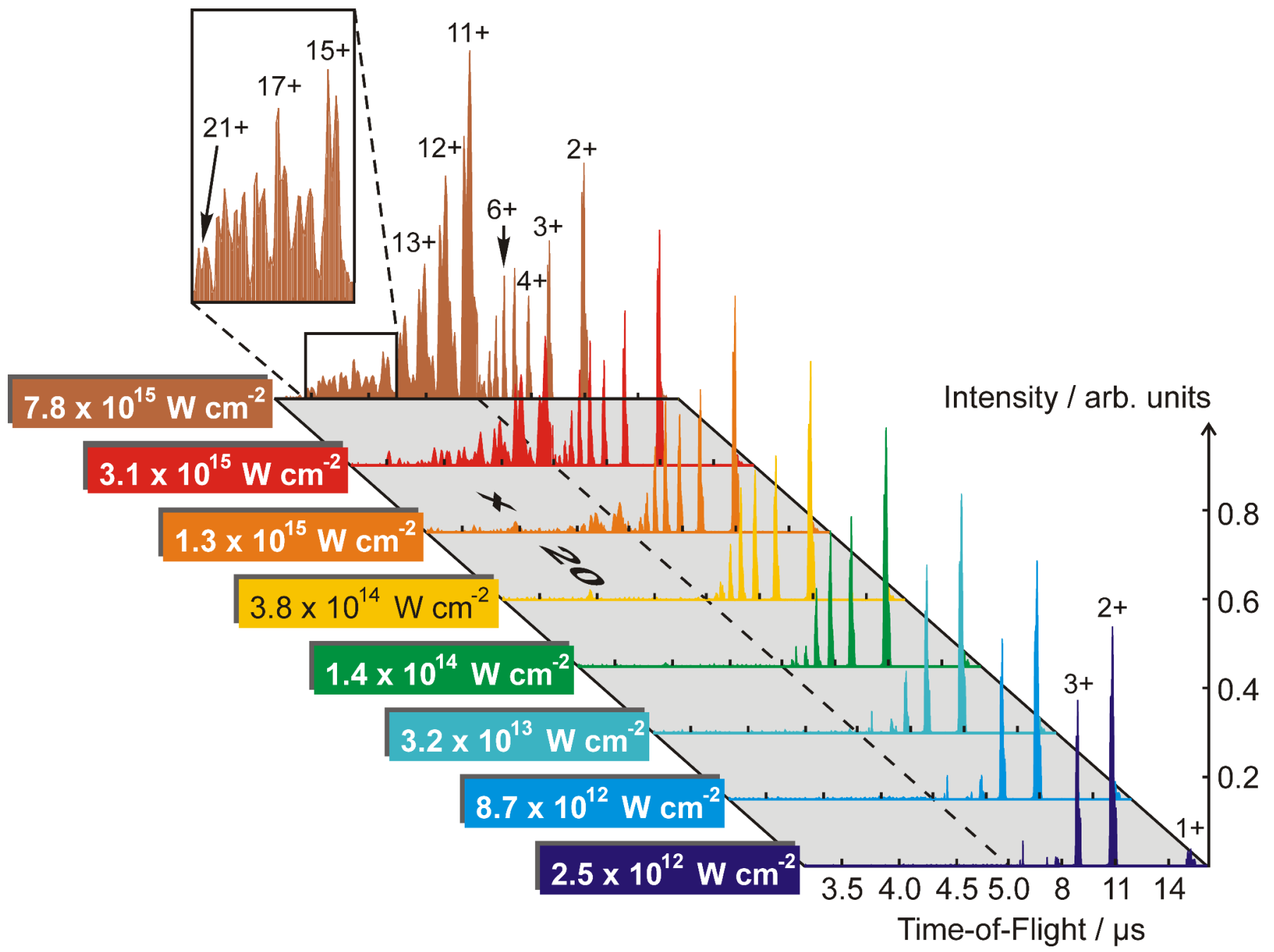

Figure 7 (Fig07_XeTOF.png) 


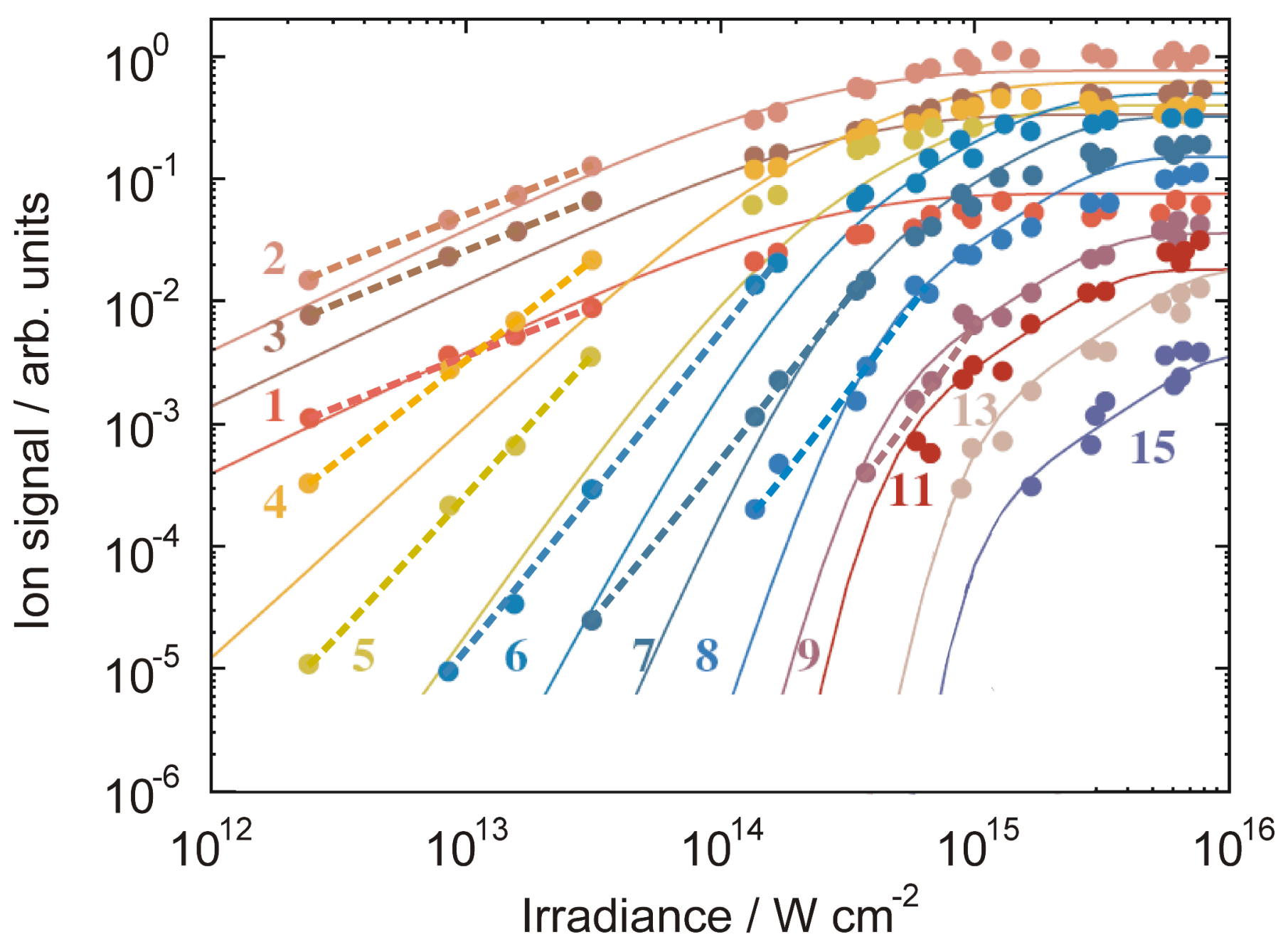

Figure 8 (Fig08_XeIntDep_rev.png) 

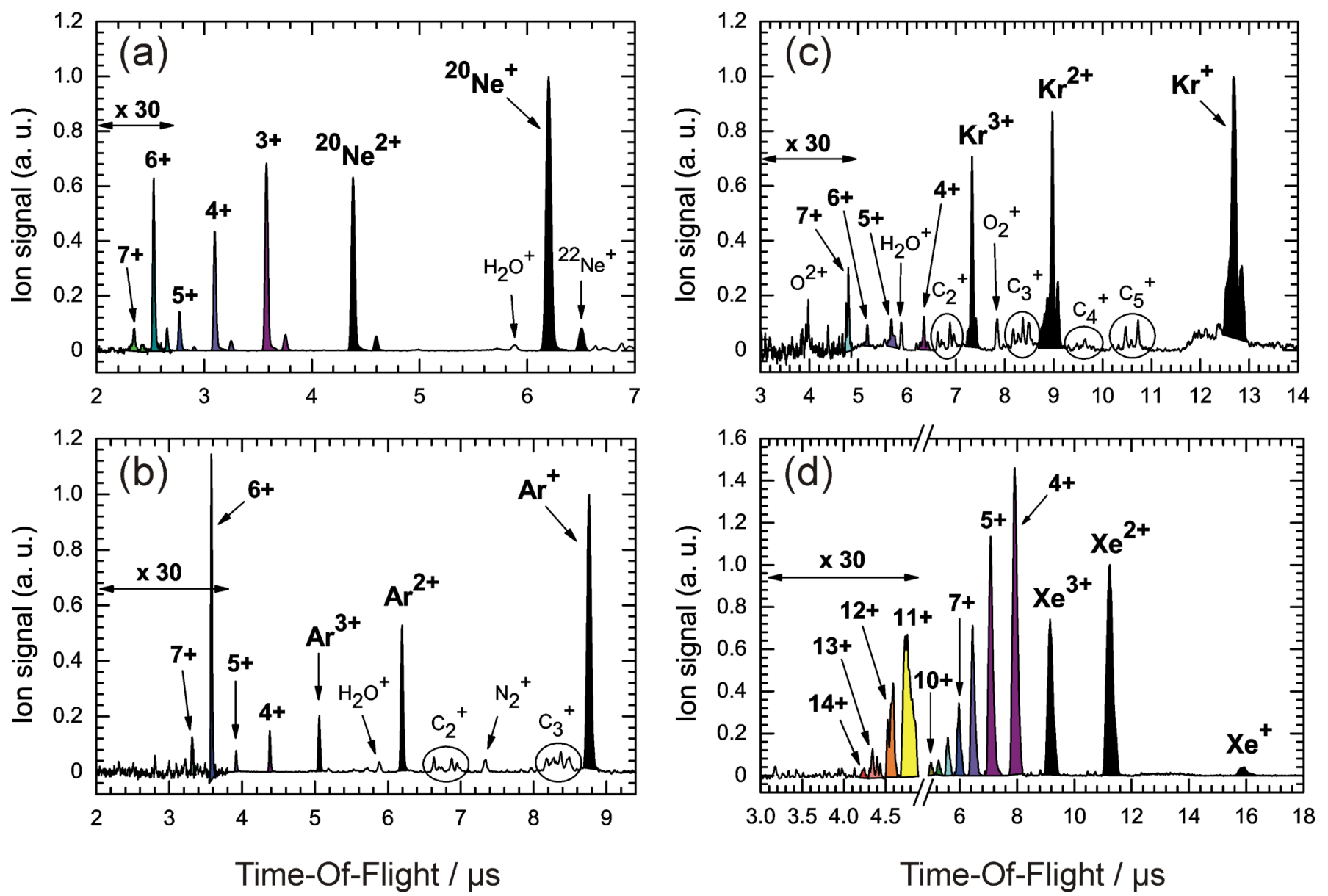

Figure 9 (Fig09_RareGasTOF.png) 